\title{
Efeito de extratos e óleos essenciais de plantas na germinação de urediniósporos de Phakopsora pachyrhizi
}

\author{
BORGES, D.I.1*; ALVES, E.2; MORAES, M.B.de²; OLIVEIRA, D.F. ${ }^{3}$ \\ Universidade Federal de Lavras, ${ }^{1}$ Departamento de Agricultura, ${ }^{2}$ Departamento de Fitopatologia, ${ }^{3}$ Departamento de \\ Química, Campus Universitário, Caixa Postal 3037, CEP 37200-000, Lavras-Brasil *dinesborges@yahoo.com.br.
}

\begin{abstract}
RESUMO: Métodos alternativos para o controle de doenças e pragas de plantas vêm sendo pesquisados buscando causar menores danos ao ambiente e a saúde humana. Diante disso, o objetivo deste estudo foi identificar extratos de plantas e óleos essenciais com possíveis efeitos sobre Phakopsora pachyrhizi, agente etiológico da ferrugem-asiática da soja. Assim, 61 extratos de plantas da flora nativa da região do Alto Rio Grande, Minas Gerais e cinco óleos essenciais obtidos comercialmente foram utilizados com a finalidade de testar os efeitos na germinação de urediniósporos do referido fungo. Foram utilizadas placas de Petri de $6 \mathrm{~cm}$, com meio ágar-água, adicionado com $150 \mu \mathrm{L}$ de extrato de planta ou $6 \mu \mathrm{L}$ do óleo essencial misturado ao meio de cultura. Como testemunhas foram utilizadas $150 \mu \mathrm{L}$ de Tween 20 a 0,7\% e $150 \mu \mathrm{L}$ de água destilada. Após, foram adicionados $50 \mu \mathrm{L}$ de uma suspensão de urediniósporos, na concentração de $2 \mathrm{mg} .5 \mathrm{~mL}^{-1}$ e espalhados com alça de Drigalsky. As placas foram colocadas em BOD, a $23\left( \pm 2^{\circ} \mathrm{C}\right)$, por 4 horas. A germinação foi paralisada com adição de lactoglicerol. A avaliação da percentagem de esporos germinados foi realizada em microscópio de luz. Dentre os extratos vegetais testados, dez apresentaram percentual de germinação abaixo de $15 \%$, tendo os extratos obtidos das plantas Pelargonium sp., Salvia officinalis, Lavandula officinalis, Mentha pulegium e M. arvensis apresentado germinação de esporos de 3,0\%, 3,5\%, 3,0\%, 2,0\% e 4,0\%, respectivamente. Todos os óleos essenciais avaliados foram efetivos na inibição da germinação, com destaque para o óleo essencial de Caryophilus aromaticus, que apresentou percentagem de germinação de 5\%, resultando num percentual de inibição de 50,0\%.
\end{abstract}

Palavras-chave: controle alternativo de fitopatógenos, ferrugem-asiática da soja

ABSTRACT: Effect of plant extracts and essential oils on the germination of urediniospores of Phakopsora pachyrhizi. Alternative methods to control plant diseases and plagues have been studied, in order to cause less damages to the environment and human health. The purpose of this study was to identify plant extracts and essential oils as possible alternative products that affect the urediniospore germination of Phakopsora pachyrhizi, the causal agent of the Asian soybean rust. Plant extracts (61) from the native flora of the Alto Rio Grande region, State of Minas Gerais, Brazil, as well as five commercial essential oils were used with the purpose of testing their effects on the germination of urediniospores of the referred fungus. $6 \mathrm{~cm}$ Petri dishes were used, with water-agar medium, added with $150 \mu \mathrm{L}$ of plant extract or $6 \mu \mathrm{L}$ of essential oil mixed to the culture medium. As control, $150 \mu \mathrm{L}$ of Tween 20 at $0.7 \%$ and $150 \mu \mathrm{L}$ of distilled water were used. Then, $50 \mu \mathrm{L}$ of a suspension of urediniospores were added, at a concentration of $2 \mathrm{mg} \mathrm{mL}^{-1}$ and spread with Drigalsky spatula. Then, the plates were put in BOD, at $23\left( \pm 2^{\circ} \mathrm{C}\right)$, for 4 hours. Germination was paralyzed by adding lactoglicerol. The percentage of spore germination was evaluated in a light microscope. Among the vegetal extracts tested, ten of them presented a germination percentage below $15 \%$. The extracts from the Pelargonium sp., Salvia officinalis, Lavandula officinalis, Mentha pulegium and $M$. arvensis plants presented $3.0 \%, 3.5 \%, 3.0 \%, 2.0 \%$ and $4.0 \%$ of urediniospore germination, respectively. All the essential oils evaluated were effective in inhibiting germination, especially the Caryophilus aromaticus essential oil that presented $5.0 \%$ of germination, with $50.0 \%$ of inhibition.

Keywords: alternative control of plant diseases, Asian soybean rust

Recebido para publicação em 23/08/2010

Aceito para publicação em 22/08/2012

Rev. Bras. PI. Med., Campinas, v.15, n.3, p.325-331, 2013. 


\section{INTRODUÇÃO}

A rápida expansão e o grande potencial de perdas na produção fazem da ferrugem-asiática uma das mais importantes doenças da cultura da soja (Yorinori et al., 2005). O fungo, muito agressivo, pode causar rápido amarelecimento e queda prematura de folhas causando danos na formação das vagens e enchimento de grãos (Yang et al., 1991).

Devido ao grande emprego de agrotóxicos causando danos ao ambiente e com o surgimento de fitopatógenos resistentes a essas substâncias químicas, pesquisas têm sido realizadas com a finalidade de identificar formas alternativas de controle de pragas e doenças de plantas procurando reduzir os impactos ambientais e os riscos de intoxicação humana, causados pelo atual modelo de produção agrícola. Somando-se a isto, a produção de soja orgânica está se difundindo no Brasil e, atualmente, o país se destaca como o maior produtor mundial. Esta cadeia encontra-se sem alternativas para o controle da ferrugem uma vez que os produtos disponíveis para o controle da doença não podem ser utilizados (Medice et al., 2007).

Segundo Sholberg \& Gaunce (1995), as substâncias sintetizadas naturalmente pelas plantas, em resposta às necessidades ecológicas e de desenvolvimento, protegem-nas contra o ataque de patógeno. Assim, os extratos e óleos essenciais extraídos de plantas vêm sendo utilizados em estudos visando à redução do uso de fungicidas e com resultados promissores no controle de vários fitopatógenos.

Pesquisa realizada in vitro é o primeiro passo para identificar o potencial de produtos oriundos de plantas medicinais da flora nativa no controle de fitopatógenos. Trabalhos desenvolvidos com extrato bruto e óleo essencial têm indicado o potencial das mesmas em diversos patossistemas, por sua ação fungitóxica direta, inibindo o crescimento micelial e a germinação de esporos (Schwan-Estrada et al., 2003).

Assim, este trabalho teve por objetivo avaliar o efeito de extratos e óleos essenciais vegetais no processo de germinação de urediniósporos de $P$. pachyrhizi.

\section{MATERIAL E MÉTODOS}

Foram utilizados cinco óleos essenciais obtidos comercialmente (Tabela 1) e 61 extratos vegetais (Tabela 2) fornecidos pelo Laboratório de Produtos Naturais, coordenado pelo Prof. Denílson Ferreira de Oliveira, do Departamento de Química da Universidade Federal de Lavras. Para o preparo dos extratos, partes de tecidos vegetais (fruto, folhas, flores) foram maceradas com metanol, por 48 horas. Essa mistura foi filtrada, dando origem ao resíduo e ao filtrado. O resíduo foi imerso em mais metanol durante 48 horas, dando origem a um novo resíduo e a um novo filtrado, o qual foi adicionado ao filtrado anterior, originando solução que foi concentrada em rota-evaporador e liofilizada.

Os urediniósporos de $P$. pachyrhizi foram obtidos de plantas de soja da cultivar MG/BR 46 (Conquista), naturalmente infectadas, provenientes do campo experimental da UFLA, em Lavras, MG, coletados pelo método da batida, utilizado por Magnani Zambenedetti et al. (2007). Em seguida, foram pesados e colocados em béquer contendo água destilada e Tween 20 a $0,7 \%$, agitando por três minutos. Foi preparada a suspensão de urediniósporos na concentração de $2 \mathrm{mg}^{-} 5 \mathrm{~mL}^{-1}$ (em média, $2 \times 10^{4}$ urediniósporosmL-1).

TABELA 1. Óleos essenciais comerciais utilizados nos testes in vitro para verificar o efeito sobre a germinação de urediniósporos de Phakopsora pachyrhizi.

\begin{tabular}{cc}
\hline Nome científico & Nome comum \\
\hline Rosmarinus officinalis L. & Alecrim \\
Cymbopogun citratus (DC.) Stapf & Capim-limão \\
Eucalyptus citriodora Hook. & Eucalipto citriodora \\
Caryophilus aromaticus L. & Cravo-da-índia \\
Piper aduncum L. & Pimenta-de-macaco \\
\hline
\end{tabular}

Os extratos vegetais e os óleos essenciais foram avaliados, em seis experimentos devido ao número de tratamentos. Como testemunha foi utilizada Tween 20 a 0,7\% em cada etapa.

Para os testes de germinação foram utilizadas duas placas de Petri com $6,0 \mathrm{~cm}$ de diâmetro contendo meio ágar-água para cada tratamento. A essas, foram adicionados $150 \mu \mathrm{L}$ de extrato vegetal ou $6 \mu \mathrm{L}$ de óleo essencial incorporado ao meio de cultura e $50 \mu \mathrm{L}$ da suspensão de urediniósporos, espalhado sobre o meio de cultura, na concentração de $2 \mathrm{mg} .5 \mathrm{~mL}^{-1}$ de água destilada. Em seguida, as placas foram levadas para câmara de germinação a $23^{\circ} \mathrm{C}$ $\left( \pm 2^{\circ} \mathrm{C}\right)$, por 4 horas. Após a incubação, o processo foi paralisado utilizando-se lactoglicerol e avaliou-se a porcentagem de germinação em microscópio de luz, com aumento de 400x. Os urediniósporos foram considerados germinados quando o comprimento do tubo germinativo era duas vezes maior que 0 tamanho do esporo.

O delineamento experimental foi inteiramente casualizado. Foram utilizadas duas placas por tratamento, divididas em dois compartimentos, sendo cada compartimento constituído por uma repetição, em

Rev. Bras. PI. Med., Campinas, v.15, n.3, p.325-331, 2013. 
TABELA 2. Plantas fontes de extratos vegetais utilizados nos testes in vitro para verificar o efeito sobre a germinação de urediniósporos de Phakopsora pachyrhizi.

\begin{tabular}{|c|c|c|c|}
\hline Nome científico & Nome comum & Nome científico & Nome comum \\
\hline Ficus carica L. & Figueira & Coix lacryma-jobi L. & Lágrima-de-nossa-senhora \\
\hline Coffea arábica L & Café arábica & Jatropha curcas L. - fruto & Pinhão manso \\
\hline Achillea millefolium L. -folha & Mil-folhas & Hypericum perforatum L. & Erva-de-são-joão \\
\hline Tanacetum vulgare L. & Catinga-de-mulata & Artemísia annua L. & Artemísia \\
\hline Plantago lanceolata Hook. & Transagem & Mentha piperita L. & Hortelã-pimenta \\
\hline Ocimum basiculum L. & Manjericão & Pelargonium sp. & Gerânio \\
\hline Musa sapientum L. & Bananeira & Chenopodium ambrosioides L. & Erva-de-santa-maria \\
\hline Achillea millefolium L. - flor & Mil-folhas & Mirabilis jalapa L. & Maravilha \\
\hline Zingiber officinale Roscoe. & Gengibre & Sambucus nigra L. - folha & Sabugueiro \\
\hline Citrus limon (L.) Burm. & Limão-siciliano & Petiveria alliacea L. & Guiné \\
\hline Psidium guajava L. & Goiabeira & Porophyllum ruderale (Jacq.) Cassini & Arnica-paulista \\
\hline Mangifera indica $L$. & Mangueira & Salvia officinalis $L$. & Salvia \\
\hline Ricinus communis $L$. & Mamoneira & Jatropha curcas L. - folha & Pinhão manso \\
\hline Symphytum officinale $L$. & Confrei & Cynara scolymus L. & Alcachofra \\
\hline Mentha spicata $L$. & Hortelã-peluda & Curcuma longa $L$. & Curcuma \\
\hline Glechoma hederaceae $L$. & Hera terrestre & Ginkgo biloba & Ginkgo-biloba \\
\hline Rosmarinus officinalis $L$. & Alecrim & Annona squanosa $L$ & Fruta-do-conde \\
\hline Tropaeolum majus $L$. & Capuchinha & Centella asiática (L.) Urban. & Centela-asiática \\
\hline Ocimum gratissimum L. & Alfavaca & Urtica dióica $L$. & Urtiga \\
\hline Equisetum arvense $L$. & Cavalinha & Lavandula officinalis $L$. & Alfazema \\
\hline Tagetes patula L. - folha & Cravo-de-defunto & Hedera helix $L$. & Hera \\
\hline Jatropha curcas L. - flor & Pinhão manso & Momordica charantia $L$. & Melão-de-são-caetano \\
\hline Thymus vulgaris $L$. & Tomilho & Nicotiana tabacum L. & Tabaco \\
\hline Tagetes patula L. - flor & Cravo-de-defunto & Tetradenia riparia (Hochst.) Codd. & Mirra \\
\hline Jatropha curcas L. - flor & Pinhão manso & Momordica charantia $L$. & Melão-de-são-caetano \\
\hline Thymus vulgaris $L$. & Tomilho & Nicotiana tabacum L. & Tabaco \\
\hline Tagetes patula L. - flor & Cravo-de-defunto & Tetradenia riparia (Hochst.) Codd. & Mirra \\
\hline Mimosa pudica L. - folha & Dormideira & Mentha pulegium L. & Poejo \\
\hline Punica granatum L. & Romã & Pteridium aquilinum (L.) Kuhn. & Samambaia \\
\hline Tropaeolum majus L. & Capuchinha & Petiveria alliaceae L. A & Amansa-senhor \\
\hline Mimosa pudica L. - flor & Dormideira & Taraxacum officinale Weber ex F.H. Wigs & g. Dente-de-leão \\
\hline Sambucus nigra L. - flor & Sabugueiro & Melissa officinalis L. & Erva-cidreira \\
\hline Citrus aurantium L. & Laranja-amarga & Mentha arvensis L. & Menta \\
\hline Tília cordata Mill. & Tília & - & - \\
\hline
\end{tabular}

que 50 urediniósporos foram observados, num total de 200 urediniósporos por tratamento.

Devido à grande quantidade de valores zero de germinação, os dados foram transformados para arco-sen $\sqrt{ } \mathrm{x} / 100$, em que $\mathrm{x}$ é a porcentagem observada. As análises foram feitas no programa Sisvar (Ferreira, 2000) e as médias comparadas pelo teste de Scott-Knot, a 5\%.

RESULTADO E DISCUSSÃO
A germinação dos urediniósporos em contato com a solução contendo Tween 20 a 0,7\% variou de $10,0 \%$ a $51,5 \%$, nos seis experimentos (Tabela 3).

Em todos os experimentos conduzidos, os resultados evidenciaram grande diversidade quanto ao percentual de urediniósporos germinados, tanto para a inibição como para a estimulação da germinação. Esta variação é comum para este fungo

Rev. Bras. PI. Med., Campinas, v.15, n.3, p.325-331, 2013. 
TABELA 3. Percentagem de germinação de urediniósporos de Phakopsora pachyrhizi, nos seis experimentos, quando em contato com Tween 20 a $0,7 \%$.

\begin{tabular}{cc}
\hline Tween $\mathbf{2 0}$ a $\mathbf{0 , 7} \%$ & \% urediniósporos germinados \\
\hline Experimento I & 30,0 \\
Experimento II & 49,5 \\
Experimento III & 21,0 \\
Experimento IV & 40,0 \\
Experimento V & 51,5 \\
Experimento VI & 10,0 \\
\hline
\end{tabular}

que apresenta perda de viabilidade do esporo muito rápida (Magnani Zambenedetti et al, 2007)

No experimento I (Tabela 4), os dados de percentual de germinação dos urediniósporos variaram. Os extratos de Tanacetum vulgaris e Ocimum gratissimum apresentaram um percetual médio de inibição de $45,83 \%$, comparados com a testemunha. O extrato de flor de Achillea millefolium foi o que se destacou na redução da germinação de urediniósporos, apresentando percentual de inibição da germinação de $60,0 \%$, comparado com a testemunha Tween 20 a 0,7\%.

O percentual de germinação apresentado pelo extrato de $A$. millefolium foi variável de acordo com a parte vegetal utilizada. Quando utilizada a folha, ocorreu um estímulo da germinação, com percentual de 1,66\% comparada com a testemunha. Pode-se concluir que a substância bioativa na inibição da germinação está concentrada na flor dessa espécie, chamada de mil-folhas. Segundo Fiori et al. (2000), o extrato de folhas de $A$. millefolium foi responsável por $46,0 \%$ de inibição na germinação de esporos de Didymella bryoniae.

Outros extratos também foram testados neste experimento. $O$ extrato Equisetum arvense demonstrou efeito estimulatório, com $1,66 \%$ quando comparado com a testemunha. Os extratos de Plantago lanceolata e Ficus carica, apresentaram $15,84 \%$ de inibição da germinação, em média, seguido do extrato de Coffea arabica, com 3,34\% de inibição.

Extratos vegetais obtidos de plantas do gênero Ocimum têm sido amplamente avaliados em diversos patossistemas. Estas plantas possuem alguma substância, ainda não identificada, que é capaz de inibir a germinação de esporos de fungos.

Para o Experimento II, todos os extratos vegetais mostraram percentual de germinação inferiores a testemunha (Tabela 4). Os extratos obtidos de flor de Jatropha curcas, Citrus aurantium e de Thymus vulgaris apresentaram valores médios de inibição da germinação de urediniósporos quando
TABELA 4. Percentagem de germinação de urediniósporos de Phakopsora pachyrhizi, sob o efeito de extratos vegetais. UFLA, Lavras, MG, 2007.

\begin{tabular}{|c|c|c|}
\hline $\begin{array}{c}\text { Extrato } \\
\text { vegetal - nome } \\
\text { científico }\end{array}$ & $\begin{array}{l}\text { \% } \\
\text { ediniósporos } \\
\text { yerminados }\end{array}$ & $\begin{array}{c}\% \text { de inibição } \\
\text { comparada } \\
\text { com o do } \\
\text { Tween } 20 \text { a } \\
0,7 \%\end{array}$ \\
\hline \multicolumn{3}{|c|}{ Experimento I } \\
\hline Citrus limon & 22,5 & 25,0 \\
\hline Tanacetum vulgaris & 16,5 & 45,0 \\
\hline Ocimum gratissimum & 16,0 & 46,66 \\
\hline Ocimum basiculum & 14,0 & 53,33 \\
\hline Achillea millefolium flor & 12,0 & 60,0 \\
\hline \multicolumn{3}{|c|}{ Experimento II } \\
\hline Jatropha curcas flor & 17,0 & 65,65 \\
\hline Citrus aurantium & 16,0 & 67,67 \\
\hline Thymus vulgaris & 14,0 & 71,71 \\
\hline Tagetes sp. flor & 7,5 & 84,84 \\
\hline \multicolumn{3}{|c|}{ Experimento III } \\
\hline Mangifera indica & 32,0 & $+52,38$ \\
\hline Musa sapientum & 31,5 & $+50,0$ \\
\hline Zingiber officinale & 30,0 & $+42,85$ \\
\hline Cynara scolymus & 55,0 & $+37,50$ \\
\hline Rosmarinus officinalis & 20,0 & 4,76 \\
\hline Glechoma hederaceae & 20,0 & 4,76 \\
\hline Symphytum officinale & 19,5 & 7,14 \\
\hline Tropaeolum majus folha & 15,0 & 28,57 \\
\hline \multicolumn{3}{|c|}{ Experimento VI } \\
\hline Tília cordata & 75,0 & $+87,50$ \\
\hline Sambucus nigra folha & 69,5 & $+73,75$ \\
\hline Jatropha curcas fruto & 69,0 & $+72,50$ \\
\hline Coix lacryma-jobi & 67,5 & $+67,50$ \\
\hline Artemísia annua & 10,5 & 73,75 \\
\hline Salvia officinalis & 3,5 & 91,25 \\
\hline Pelargonium sp. & 3,0 & 92,50 \\
\hline \multicolumn{3}{|c|}{ Experimento $\mathrm{V}$} \\
\hline Pteridium aquilinum & 76,5 & $+48,54$ \\
\hline Taraxacum officinale & 59,0 & $+14,56$ \\
\hline Momordica charantia & 57,5 & $+11,65$ \\
\hline Lavandula officinalis & 4,0 & 92,23 \\
\hline Mentha pulegium & 3,0 & 94,17 \\
\hline Mentha arvensis & 2,0 & 96,11 \\
\hline \multicolumn{3}{|c|}{ Experimento VI } \\
\hline Urtica dióica & 46,0 & $+360,0$ \\
\hline Melissa officinalis & 25,5 & $+155,0$ \\
\hline Eucalyptus citriodora ${ }^{o e}$ & 8,0 & 20,0 \\
\hline Piper aducumoe & 6,5 & 35,0 \\
\hline Rosmarinus officinalis ${ }^{o e}$ & 5,5 & 45,0 \\
\hline Cymbopogun citratus ${ }^{\circ e}$ & 5,5 & 45,0 \\
\hline Caryophilus aromaticus ${ }^{o e}$ & 5,0 & 50,0 \\
\hline
\end{tabular}

+ corresponde ao estimulo à germinação de urediniósporos. oe óleo essencial 
comparados com a testemunha de $65,65 \%, 67,67 \%$ e $71,71 \%$, respectivamente.

Neste experimento foi avaliado o efeito de duas espécies de plantas tomando-se a flor e a folha para a produção dos extratos. Já para os extratos de Tagetes sp., os resultados foram diferentes sendo mais efetivo dentre todos, aquele obtido da flor com $7,5 \%$ de germinação, indicando que a inibição da germinação de urediniósporos foi de $84,84 \%$ em relação a testemunha Tween 20 a 0,7\%. Já para os extratos obtidos de flor e folha de Mimosa pudica, o percentual de inibição foi de $47,47 \%$ e $58,58 \%$, respectivamente.

Os extratos de flor de Tropaeolum majus, flor de Sambucus nigra e de Punica granatum, outros avaliados, apresentaram 43,43\% em média, de inibição da germinação quando comparado com a testemunha.

No experimento III, o percentual obtido com a utilização dos extratos vegetais foi variável, desde extratos que apresentaram um baixo percentual de germinação até os que foram capazes de estimular a germinação de urediniósporos.

$\mathrm{O}$ extrato de Mangifera indica apresentou característica de estímulo à germinação de urediniósporos, conferindo $52,38 \%$ a mais comparando-se com a testemunha. Em seguida, os extratos de Musa sapientum, Zingiber officinale, com $50,0 \%$ e $42,85 \%$ de estímulo, respectivamente (Tabela 4). Com resposta semelhante, os extratos de Psidium guajava e Ricinus communis, ambos apresentaram $28,57 \%$ de estímulo à germinação. Esta informação pode ser importante para o desenvolvimento de novos estudos com este fungo que tem problemas de germinação, pois o uso destes extratos pode vir a potencializar a germinação quando de inoculações em plantas de soja e também indica que na verdade o esporo não estava inviável, mas apenas dormente.

Os extratos obtidos de Rosmarinus officinalis, Glechoma hederaceae, Symphytum officinale e de Tropaeolum majus, foram os que demonstraram efeito de inibição da germinação dos urediniósporos neste experimento, com destaque para o último, que apresentou $15,0 \%$ de germinação, indicando inibição, quando comparado com a testemunha, de $28,58 \%$.

O tratamento testemunha apresentou baixo percentual germinativo $(21,0 \%)$, possivelmente devido a algum fator intrínseco aos urediniósporos.

O efeito do extrato de Symphytum officinale, popularmente chamada de confrei, foi avaliado na germinação de outros fungos. Karavaev et al. (2001) observaram que tanto este como o extrato obtido de Bauhinia sp. (pata-de-vaca) foram capazes de inibir a germinação de conídios de Erysiphe graminis e urediniósporos de Puccinia graminis, respectivamente agentes causais do oídio e ferrugem-do-colmo em trigo. Salustiano et al. (2006), trabalhando com o mesmo fungo e ainda outros dois fungos agentes causais de ferrugem o Hemileia vastatrix e Cerotelium fici, utilizaram extrato de candeia (Eremanthus erythropappus) com extração alcoólica, aquosa e o chá da folha, na concentração de $10 \%$, bem como o óleo essencial, a 1\%. Após períodos de incubação de 4, 24 e 48 horas, respectivamente, todos os extratos e o óleo essencial foram capazes de inibir em 100\% a germinação dos esporos.

No experimento IV, os dados de germinação foram semelhantes ao apresentado pelo experimento III, com resultados de estímulo e de inibição da germinação de urediniósporos.

Alguns extratos vegetais apresentaram um efeito de estimulo na germinação com destaque para o extrato de Tília cordata com $87,50 \%$ de estímulo comparado à testemunha. Além deste, outros extratos apresentaram tal efeito, como os extratos de folha de Sambucus nigra, frutos de Jatropha curcas, Coix lacryma-jobi e de Cunara scolymus, com estímulo de $73,75 \%, 72,50 \%$, $67,50 \%$ e $37,50 \%$, respectivamente, comparados a testemunha. Outros extratos com o mesmo efeito foram detectados. Os obtidos de Hypericum perforatum e de Mirabilis jarapa apresentam uma média de $17,50 \%$ de estímulo.

Várias substâncias podem regular a germinação de urediniósporos incluindo endógenos de auto-inibição química e substâncias que estimulam a germinação (Lucas \& Knight, 1987; French, 1992; Griffin, 1994). Tessmann \& Dianese (2002) trabalharam com o extrato obtido de folhas de jambeiro (Syzygium jambos). Frações do extrato foram purificadas por meio de cromatografia de coluna e foram testadas sobre a germinação de urediniósporos de Puccinia psidii. A fração diluída com n-hexano $100 \%$ aumentou a germinação em até $88 \%$ comparado à testemunha com somente óleo mineral. Assim, estes como também outros resultados podem contribuir para o melhor entendimento do processo de infecção das ferrugens nas plantas hospedeiras. No caso de P. pachyrhizi, substâncias que estimulam a germinação podem auxiliar na compreensão do complexo processo de germinação desse patógeno.

Por outro lado, neste experimento efeito inibitório da germinação foi detectado com o uso dos extratos de Artemísia annua, Salvia officinalis e Pelargonium sp. Nesses extratos foram obtidos $10,5 \%, 3,5 \%$ e $3,0 \%$ de germinação de urediniósporos, com inibição média de $73,75 \%$, $91,25 \%$ e $92,50 \%$, respectivamente, quando comparado com a testemunha.

Outros extratos testados também

Rev. Bras. PI. Med., Campinas, v.15, n.3, p.325-331, 2013. 
apresentaram inibição da germinação quando comparados com a testemunha. Nos extratos de Porophyllum ruderale e de Petiveria alliacea, o percentual de inibição foi de $30,0 \%$ e $41,25 \%$, respectivamente. No extratos de folha de Jatropha curcas a inibição foi de $41,25 \%$ e para os extratos de Chenopodium ambrosioides e de Mentha piperita, o percentual médio foi de $54,25 \%$.

No Experimento V, os dados apresentaram ampla variação tendo diversos extratos sidos responsáveis por causar inibição e outros proporcionado algum estímulo para a germinação dos urediniósporos (Tabela 4).

Dentre os extratos testados, o de Pteridium aquilinum apresentou percentual de $76,5 \%$ de germinação, o que representa $48,54 \%$ de estímulo na germinação dos urediniósporos comparado com a testemunha. Outros extratos vegetais também apresentaram efeito estimulatório, como os obtidos de Taraxacum officinale e Momordica charantia, com $14,56 \%$ e $11,65 \%$, respectivamente.

A maioria dos extratos testados foi promissora para a inibição da germinação dos urediniósporos. Dentre esses, merecem destaque os extratos de Lavandula officinalis, Mentha pulegium e de M.arvensis com percentuais de $92,23 \%$, $94,17 \%$ e $96,11 \%$ de inibição. Esses altos valores encontrados foram importantes para a seleção desses extratos para testes in vivo.

Com o mesmo efeito inibitório, outros extratos apresentaram resultados satisfatórios. Os extratos de Petiveria alliaceae e de Nicotiana tabacum resultaram em $7,77 \%$ e $19,42 \%$ de inibição respectivamente, quando comparado com a testemunha. Em média, os extratos de Hedera helix, Tetradenia riparia e de Anonna aquosa, apresentaram percentual de inibição de $39,16 \%$ e os extratos de Centella asiática e de Curcuma longa, com $54,37 \%$.

A cúrcuma (Curcuma longa) tem sido amplamente empregada em diversas finalidades. Neste experimento, o extrato dessa planta mostrouse eficiente no que diz respeito ao controle de germinação de esporos de fungos com um percentual de $69,91 \%$ de inibição, quando comparado com a testemunha. Balbi-Peña et al. (2006), utilizando extratos de cúrcuma e da curcumina (substância extraída da cúrcuma), observaram que os extratos brutos não autoclavados promoveram a inibição da germinação de esporos de Alternaria solani de até $15 \%$, o que não ocorreu com os extratos autoclavados, indicando a presença de algum composto termolábil.

No Experimento $\mathrm{VI}$, foram testados os óleos essências e dois extratos vegetais remanescentes. Os extratos vegetais apresentaram efeito estimulatório da germinação quando comparados com a testemunha. O percentual de estímulo para o extrato de Melissa officinalis foi alto, com $360 \%$, já para o extrato de Urtica dióica, o estímulo foi um pouco menor, com 155,0\%. (Tabela 4).

Para os óleos essenciais utilizados, todos apresentaram resultados na inibição da germinação dos urediniósporos, com destaque para o óleo essencial de Caryophilus aromaticus, que apresentou 50,0\% de inibição (Tabela 9).

Para diversos fungos, o óleo essencial do gênero Piper sp. é efetivo na inibição de esporos. A espécie avaliada neste experimento foi Piper aduncum, que apresentou bom percentual de inibição $(35,0 \%)$.

Silva \& Bastos (2007) verificaram a atividade do óleo essencial de 10 espécies de Piper. A inibição total do crescimento micelial de Crinipellis perniciosa foi verificado com o óleo de $P$. callosum $\left(0,75 \mu \mathrm{LmL}^{-1}\right)$ e $P$. marginatum var. anisatum $\left(1 \mu \mathrm{LLL}^{-1}\right)$ e para Phytopthora capsici, com o uso do óleo de $P$. callosum $\left(0,75 \mu \mathrm{LmL}^{-1}\right)$. Para P. palmivora, os óleos de P. callosum $(1 \mu \mathrm{LmL}-$ 1) e $P$. enckea $\left(1 \mu \mathrm{LmL}^{-1}\right)$ mostraram mais efetivos. Bastos \& Albuquerque (2004) identificaram que o óleo essencial de $P$. aduncum causou inibição total da germinação de conídios de Colletotrichum musae em concentrações superiores a $0,01 \%(\mathrm{v} / \mathrm{v})$. Essa mesma planta e também Lippia sidoides, tanto na forma de extrato como o óleo essencial, foram efetivas para a inibição do fungo Lasidiplodia theobromae (Mota et al., 2002).

O óleo essencial de E. citriodora também apresentou potencial de inibição, com percentual de $20,0 \%$. De acordo com Bonaldo et al. (2007), quando o extrato aquoso a $1 \%$ dessa espécie de planta foi utilizado, a germinação dos urediniósporos de $P$. pachyrhizi foi de apenas 5\%. Em outro trabalho, Bonaldo et al. (2004) verificaram que a germinação de conídios de Colletotrichum lagenaria foi inibida em mais de $90 \%$ na presença de extratos autoclavados de E.citriodora, enquanto que, para o extrato não autoclavado, a inibição máxima foi de $75 \%$.

$\mathrm{Na}$ literatura, são citados vários trabalhos com o objetivo de avaliar o efeito de extratos vegetais e óleos essenciais na germinação de diversos fungos, mas, para o agente causal da ferrugem-asiática da soja, $P$. pachyrhizi, pouco foi encontrado.

Medice et al. (2007) observaram que tanto o óleo essencial de citronela como os óleos essenciais de E. citriodora, Azadirachta indica (nim) e Thymus vulgaris (tomilho), nas concentrações $0,5 \%, 1,1 \%$ e $0,3 \%$, foram capazes de inibir em $100 \%$ a germinação de urediniósporos $P$. pachyrhizi.

Neste experimento, observou-se que a atividade inibitória do óleo de E. citriodora foi alta, mesmo na concentração de $0,1 \%$. 


\section{CONCLUSÃO}

Dentre os extratos vegetais testados, dez apresentaram percentagem de germinação de urediniósporos abaixo de 15\%, destacandose os extratos de Mentha arvensis, M. pulegium, Lavandula officinalis, Pelargonium sp. e Salvia officinalis, podendo ser considerados promissores para o controle do patógeno estudado.

Outros extratos vegetais apresentaram estimulo da germinação. Foram eles: os extratos de Tília cordata, folha de Sambucus nigra, fruto de Jatropha curcas e Coix lacryma-jobi, os quais poderiam ter alguma aplicação em estudos da biologia do patógeno.

Os óleos essenciais avaliados apresentaram resultados promissores, tendo os óleos essenciais de Caryophilus aromaticus, Cymbopogun citratus e de Rosmarinus officinalis sido os destaques.

\section{REFERÊNCIA}

BALBI-PEÑA, M.I. et al. Controle de Alternaria solani em tomateiro por extratos de Curcuma longa e curcumina: I. avaliação in vitro. Fitopatologia Brasileira, v.31, n.3, p.310-14,2006.

BASTOS, C.N.; ALBUQUERQUE, P.S.B. Efeito do óleo Piper aduncum no controle em pós-colheita de Colletotricum musae em banana. Fitopatologia Brasileira, v.29, n.5, p.555-7,2004.

BONALDO, S.M. et al. Fungitoxicidade, atividade elicitora de fitoalexinas e proteção de pepino contra Colletotrichum lagenarium, pelo extrato aquoso de Eucalyptus citriodora. Fitopatologia Brasileira, v.29, n.2, p.128-134, 2004.

FERREIRA, D.F. Análise estatística por meio do SISVAR para Windows versão 4.0. In: REUNIÃO ANNUAL DA REGIÃO BRASILEIRA DA SOCIEDADE INTERNACIONAL DE BIOMETRIA, 45, 2000, São Carlos, SP. Programa e Resumos...São Carlos: UFScar, 2000. p.235.

FIORI, A.C.G. et al. Antifungal activity of leaf extracts and essential oils of some medicinal plants against Didymella bryoniae. Journal of Phytopathology, v.148, n.7/8, p.483-488, 2000.

FRENCH, R.C. Volatile chemical germination stimulators of rust and other fungal spores. Mycologia, v.84, p.277288, 1992.
GRIFFIN, D. H. Fungal physiology. $2^{\text {th }}$ ed. New York: Wiley-Liss, 1994.

KARAVAEV, V.A. et al. Antifungal activity of aqueous extracts of the leaves of cowparsnip and comfrey. Biology Bulletin, v.28, n.4, p.365-370, 2001.

LUCAS, J.; KNIGHTS, I. Spores on leaves: endogenous and exogenous control of development. In: PEGG, G.F.; AYRES, P.G. (Eds.). Fungalinfection of plants. Cambridge: Cambridge University, 1987. p.45-59;

MAGNANI ZAMBENEDETTI, E.B. et al. Gerrminação de urediniósporos de Phakopsora pachyrhizi em diferentes métodos de armazenamento. Summa Phytopathologica,v.33, n.1, p.83-85, 2007.

MEDICE, R. et al. Óleos essenciais no controle da ferrugem asiática da soja Phakopsora pachyrhizi Syd. \& P. Syd. Ciência e Agrotecnologia, v.31, n.1, p.8390, 2007.

MESQUINI, R.M. et al. Efeito de produtos naturais na indução de fitoalexinas em cotiledones de soja e na germinação de urediniósporos de Phakopsora pachyrhizi.Revista Brasileira de Agroecologia (Online), v.2, p.1091-94, 2007.

MOTA, J.C.O. et al. Efeito de Extratos e Óleos essenciais de Plantas Medicinais no controle in vitro de Lasiodiplodia theobromae.Fitopatología Venezolana, v.15, p.1-6, 2002;

SALUSTIANO, M.E. et al. Extratos de candeia (Eremanthus erythopappus (DC) MacLeish) na inibição in vitro de Cylindrocladium scoparium e quatro gêneros de uredinales. Cerne, v.12, n.2, p.189-193, 2006.

SCHWAN-ESTRADA, K.R.F.; STANGARLIN, J.R.; CRUZ, M.E.S. Uso de plantas medicinais no controle de doenças de plantas. Fitopatologia Brasileira, v.28, p.54-6, 2003. Suplemento;

SHOLBERG, P.L.; GAUNCE, A.P. Fumigation of fruit with acetic acid to prevent postharvest decay. Horticultural Science, v.30, p.1271-5, 1995.

SILVA, D.M.; BASTOS, C.N. Atividade antifúngica de óleos essenciais de espécies de Piper sobre Crinipellis perniciosa, Phytophthora palmivora e Phytophthora capsici. Fitopatologia Brasileira, v.32, n.2, p.26-8, 2007.

TESSMANN, D.J.; DIANESE, J. Hentriacontano: um hidrocarboneto obtido de folhas de Syzygium jambos com efeito estimulante sobre a germinação de urediniósporos de Puccinia psidii. Fitopatologia Brasileira, v.27, n.5, p.538-42, 2002.

YANG, X.B. et al. Development of yield loss models in relation to reductions of components of soybeans infected with Phakopsora pachyrhizi. Phytopathology, v.81, p.1420-6, 1991.

YORINORI, J.T. et al. Epidemics of soybean rust (Phakopsora pachyrhizi) in Brazil and Paraguay from 2001 to 2003. Plant Disease, v.89, p.675-7, 2005. 